\title{
Alterações ultraestruturais nas vilosidades do jejuno de equinos após distensão
}

\author{
[Ultraestructural alterations in the equine jejune villi after distension] \\ R.S. Costa ${ }^{1}$, G.M. Pagliosa ${ }^{2}$, G.E.S. Alves ${ }^{3}$, J.J.M. Rio Tinto ${ }^{4}$, A.C. Alessi ${ }^{5}$, R.R. Faleiros ${ }^{3}$ \\ ${ }^{1}$ Aluna de graduação - EV-UFMG - Belo Horizonte, MG \\ ${ }^{2}$ Aluna de pós-graduação - EV-UFMG - Belo Horizonte, MG \\ ${ }^{3}$ Escola de Veterinária - UFMG - Belo Horizonte, MG \\ ${ }^{4}$ Pontifícia Universidade Católica - Betim, MG \\ ${ }^{5}$ Faculdade de Ciências Agrárias e Veterinárias - UNESP - Jaboticabal, SP
}

\begin{abstract}
RESUMO
O objetivo do presente estudo foi analisar as alterações ultraestruturais nas vilosidades do jejuno de seis equinos submetidos à distensão intraluminal com solução salina. A pressão intraluminal foi mantida em $25 \mathrm{~cm}$ de água durante duas horas. As amostras de mucosa intestinal colhidas às: zero hora; duas horas de distensão; e duas horas e $12 \mathrm{~h}$ de descompressão foram analisadas por meio de microscopia eletrônica de varredura. Avaliaram-se a área e o perímetro das vilosidades e sua densidade, usando-se um programa computacional de análise de imagens (Image J). A distensão luminal promoveu aumentos da área e do perímetro das vilosidades intestinais. Essa alteração ultraestrutural ocorreu somente $12 \mathrm{~h}$ após a descompressão e considerou-se que a provável causa seria o edema promovido por aumento da permeabilidade vascular decorrente de um processo de isquemia e reperfusão da mucosa intestinal. Concluiu-se que a distensão intraluminal do jejuno equino promoveu, tardiamente, aumento das dimensões das vilosidades intestinais.
\end{abstract}

Palavras-chave: equino, distensão intestinal, isquemia, reperfusão, vilosidade

\begin{abstract}
The ultra-structural alterations in the equine jejune villi caused by intraluminal distension were analyzed. Six animals had a jejunum segment distended with saline solution. The intraluminal pressure was maintained in $25 \mathrm{~cm}$ of water for $2 \mathrm{~h}$. The mucosal samples collected ( $0 \mathrm{~h}, 2 \mathrm{~h}$ of distension, $2 \mathrm{~h}$ and $12 \mathrm{~h}$ of decompression) were analyzed with scan electronic microscopy (SEM). The area and the perimeter of the villi, as well as their density, were analyzed using a specific software for image analysis (Image J). The luminal distention and decompression induced an increase on villi dimensions (area and perimeter). This ultrastructural alteration occurred just $12 \mathrm{~h}$ after decompression, possibily due to edema caused by the increase of vascular permeability resulting from ischemia and reperfusion of the intestine mucosa. The intraluminal distension of the equine jejune caused a delayed increase of the intestine villi dimensions.
\end{abstract}

Keywords: equine, intestinal distension, ischemia, reperfusion, villus

\section{INTRODUÇÃO}

A síndrome cólica é um distúrbio que causa dor abdominal e que pode acometer qualquer órgão da cavidade abdominal. É uma das maiores causas de óbito em equinos (Thomassian, 1990). Essa enfermidade causa prejuízos para os criadores, devido ao custo do tratamento e ao número elevado de vítimas fatais. Dos diversos fenômenos que cursam com a síndrome cólica, destacam-se as obstruções que interrompem o fluxo da ingesta e acarretam uma distensão do lúmen intestinal. A distensão luminal tem sido associada a complicações graves como aderências, íleo paralítico e óbito.

Ainda não são conhecidos todos os mecanismos pelos quais a distensão promove tais complicações. No intestino delgado, estudos têm sugerido que a interrupção da irrigação das vilosidades seria um fator importante na ocorrência dessas lesões (White, 1990). Segundo

Recebido em 18 de março de 2009 
tais estudos, a distensão luminal acarretaria isquemia ao obstruir as drenagens linfática e venosa das vilosidades, resultando em edema trasmural e inflamação. Esses efeitos podem ser agravados mesmo após a restauração da irrigação normal, caracterizando a injúria de reperfusão. Alguns autores consideraram que as lesões celulares, observadas durante a reperfusão, podem ser mais graves do que as próprias lesões causadas pela isquemia (Forsyth e Guilford, 1995).

O objetivo do presente estudo foi analisar as alterações ultraestruturais ocorridas nas vilosidades do jejuno equino submetido à distensão intraluminal (DIL). A hipótese foi que a distensão intraluminal por duas horas promoveria alterações na área, no perímetro e na densidade das vilosidades jejunais e que essas alterações se agravariam nas primeiras horas após a descompressão.

\section{MATERIAL E MÉTODOS}

Foram utilizados seis equinos sem raça definida, com idade variando de quatro a 12 anos e escore corporal entre 3 e 4 (escala de 1 a 5) (Speirs, 1997). Os equinos foram vermifugados e mantidos por 15 dias em regime alimentar padronizado com feno de capim coast-cross e ração comercial com $10 \%$ de proteína. Antes de submetidos ao ato cirúrgico, os animais foram pesados e avaliados por meio de exames clínicos e laboratoriais, sendo considerados hígidos e aptos para o experimento.

O projeto CVZ 23704, protocolado com o $\mathrm{n}^{\circ}$ 144/04, foi aprovado pelo Comitê de Ética em Experimentação Animal (CETEA/UFMG) em 05/10/2005.

Após jejum de $12 \mathrm{~h}$, os animais foram prémedicados com midazolam (Dormire. Cristália Produtos Químicos Farmacêuticos Ltda. Itapira, SP) $(0,15 \mathrm{mg} / \mathrm{kg}$, IV) e éter gliceril guaiacol (Éter gliceril guaiacol. Henryfarma. São Paulo, SP) $(100 \mathrm{mg} / \mathrm{kg}, \mathrm{IV})$ e, após alcançar decúbito lateral, foram intubados pelo método de rotina. A indução e manutenção anestésicas foram feitas por meio de aparelho de anestesia inalatória, com vaporizador compensado em circuito semifechado. Foi utilizado o isofluorano (Forane. Cristália Produtos Químicos Farmacêuticos Ltda. Itapira, SP) volatilizado em $15 \mathrm{ml} / \mathrm{kg} / \mathrm{min}$ de oxigênio via sonda orotraqueal. Após a indução, foi administrada solução de Ringer lactato associada à dobutamina (Dobutrex. Eurofarma. São Paulo, SP) (1 a $5 \mathrm{mcg} / \mathrm{kg} / \mathrm{min})$, com o objetivo de manter a pressão arterial média entre 70 e $100 \mathrm{mmHg}$. A concentração de $\mathrm{CO}_{2}$ ao final da expiração $\left(\mathrm{ETCO}_{2}\right)$ foi avaliada por capnografia e mantida entre 35 e $50 \mathrm{~mm}$ de $\mathrm{Hg}$ por respiração controlada.

Procedeu-se a uma celiotomia mediana préumbilical de aproximadamente $25 \mathrm{~cm}$, por onde foi feita a exposição do jejuno. No jejuno foi demarcado um segmento de $40 \mathrm{~cm}$ a cerca de $2 \mathrm{~m}$ da transição para o íleo, e esse segmento foi submetido ao modelo de obstrução com distensão intraluminal conforme modelo previamente descrito (Dabareiner et al., 2001). Para promover distensão, utilizou-se injeção intraluminal de solução salina $(\mathrm{NaCl} 0,9 \%)$ no segmento previamente isolado por ligaduras completas murais. A solução foi injetada via sonda (do tipo uretral $\mathrm{n}^{\circ} 8$ ), introduzida no lume através de enterotomia em segmento adjacente. Essa sonda estava acoplada externamente ao recipiente contendo a solução e a um manômetro aneroide por meio de uma torneira de três vias. A pressão no lume intestinal foi mantida em $25 \mathrm{~cm}$ de água, por meio da injeção ou retirada de solução salina durante todo o período de obstrução.

O segmento experimental foi mantido distendido por duas horas. Após esse período, as ligaduras e a sonda foram retiradas, e os animais mantidos em anestesia por um período mínimo de duas horas para que ocorresse a reperfusão do segmento. A recuperação anestésica foi, então, permitida. Imediatamente ao final da anestesia, os animais receberam buprenorfina (Temgesic. Indústria Química e Farmacêutica Schering Plough. Rio de Janeiro, RJ) $(0,005 \mathrm{mg} / \mathrm{kg}$, IM) com intuito de manter a analgesia. Doze horas após a desobstrução intestinal, os animais foram sedados com xilazina (Rompum. Bayer. São Paulo, SP) (1mg/kg, IV) e acepromazina (Acepran. Univet Indústria Veterinária S.A. São Paulo, SP) $(0,1 \mathrm{mg} / \mathrm{kg}$, IV). A anestesia geral foi induzida com tiopental (Tiopental. Cristália Produtos Químicos e Farmacêuticos Ltda. Itapira, SP) $(3 \mathrm{mg} / \mathrm{kg})$ administrado na artéria carótida e procedeu-se, então, à eutanásia por infusão intravenosa de solução saturada de sulfato de magnésio. 
Amostras da parede intestinal foram colhidas antes, ao final da distensão e após duas e $12 \mathrm{~h}$ de descompressão. Essas amostras intestinais foram constituídas de fragmentos com aproximadamente $2 \times 5 \mathrm{~cm}$, colhidos na borda antimesentérica, por meio de uma incisão transversal com o bisturi incidindo todos os planos da alça intestinal. Depois de colhidas, as amostras foram imediatamente fixadas em solução de glutaraldeído tamponado e armazenadas a $4^{\circ} \mathrm{C}$, para a realização do processamento de rotina para microscopia eletrônica de varredura.

Por ocasião da análise, as amostras armazenadas em glutaraldeído foram cortadas em fragmentos com aproximadamente $16 \mathrm{~mm}^{2}$, tratadas com tetróxido de ósmio (2\%) por duas horas e, posteriormente, desidratadas por passagens consecutivas de $15 \mathrm{~min}$ em concentrações crescentes de álcool etílico. Após secagem em $\mathrm{CO}_{2}$ sob pressão, por meio de secador de ponto crítico, os fragmentos foram metalizados em metalizador de fluxo iônico por período de 70 segundos, em potência de $70 \mathrm{~mA}$. A mucosa de cada amostra foi examinada em microscópico eletrônico de varredura, operando em $15 \mathrm{kV}$ em um aumento de 100 vezes, sendo as imagens registradas em papel fotossensível.

Após análise e descrição das alterações verificadas, as imagens foram digitalizadas por meio de scanner e um microcomputador, para a realização de sua

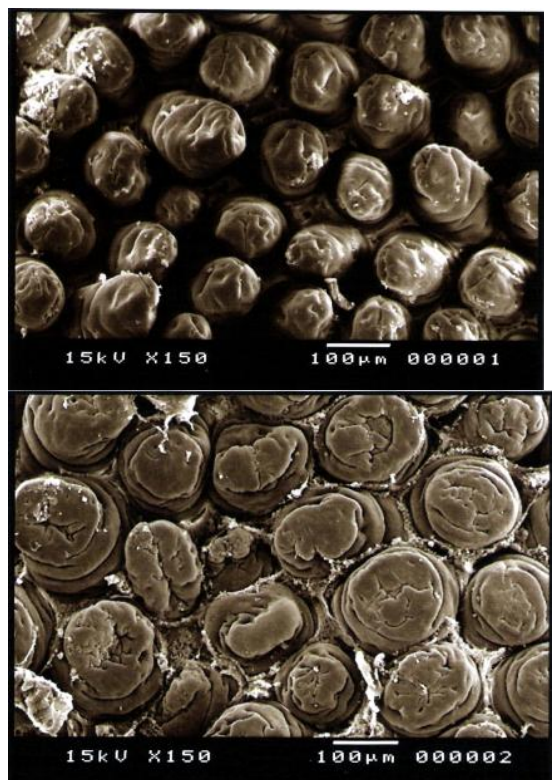

análise por software de morfometria (Image J). As análises foram realizadas pelo mesmo pesquisador. $\mathrm{Na}$ análise, foram considerados o número de vilosidades por $\mathrm{mm}^{2}$, a área e o perímetro médio das vilosidades. Para cada amostra, as medidas de área e perímetro foram obtidas de, pelo menos, 10 vilosidades.

Foram realizadas comparações específicas entre tempos. As avaliações estatísticas foram feitas por meio de análise de variância em blocos ao acaso e submetidas ao teste de Student-Newman-Keuls para comparação das médias (Sampaio, 1998). Considerou-se nível de significância de $\mathrm{P}<0,05$.

\section{RESULTADOS}

Comparando-se as características das amostras após as duas horas de distensão com as das amostras basais, verificou-se que as vilosidades apresentaram-se com aspecto achatado, com redução dos espaços entre essas estruturas. Nas primeiras duas horas após a descompressão, as vilosidades ainda apresentavam-se aplainadas em suas extremidades, mas o espaço entre elas era mais semelhante ao das amostras basais. Contudo, após $12 \mathrm{~h}$, o espaço entre as vilosidades tornou a se reduzir, sendo praticamente inexistente, e essas mostravam-se mais rugosas e avolumadas (Fig. 1).
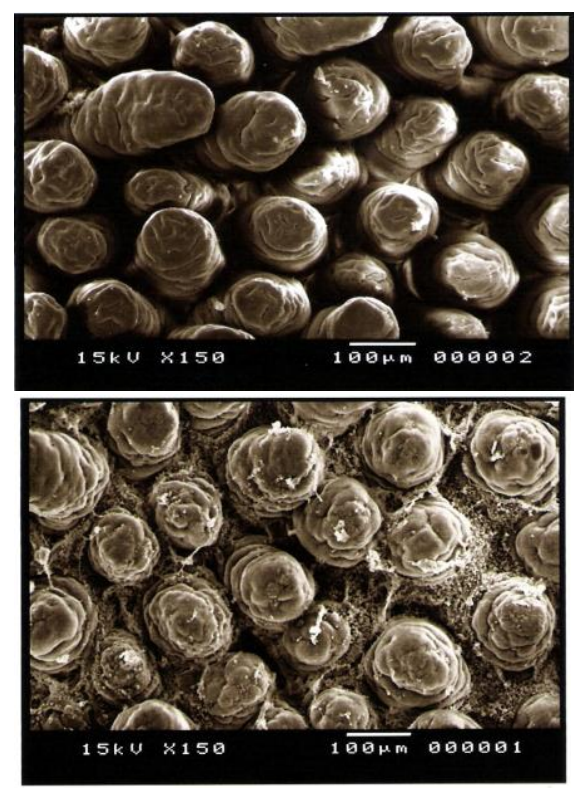

Figura 1. Eletromicrografias das vilosidades do jejuno de equinos após a distensão. Aparência ultraestrutural $(150 \mathrm{x}, 15 \mathrm{Kv})$ das vilosidades intestinais antes, após duas horas de distensão e após duas e $12 \mathrm{~h}$ de reperfusão. Após $12 \mathrm{~h}$ de descompressão, há alteração nas dimensões das vilosidades. 
Após duas horas de distensão ou duas horas de descompressão, não houve alterações significativas nas dimensões ou densidade das vilosidades em comparação com as basais. Entretanto, 12h após a descompressão, a área e o perímetro das vilosidades foram superiores $(\mathrm{P}<0,05)$ aos valores de todos os outros tempos, atingindo, respectivamente, médias $55 \%$ e $25 \%$ acima das basais. Apesar de não haver significância $(\mathrm{P}=0,07)$, nesse mesmo tempo, observou-se menor número de vilosidades por $\mathrm{mm}^{2}$. Os dados da morfometria estão contidos na Fig. 2.
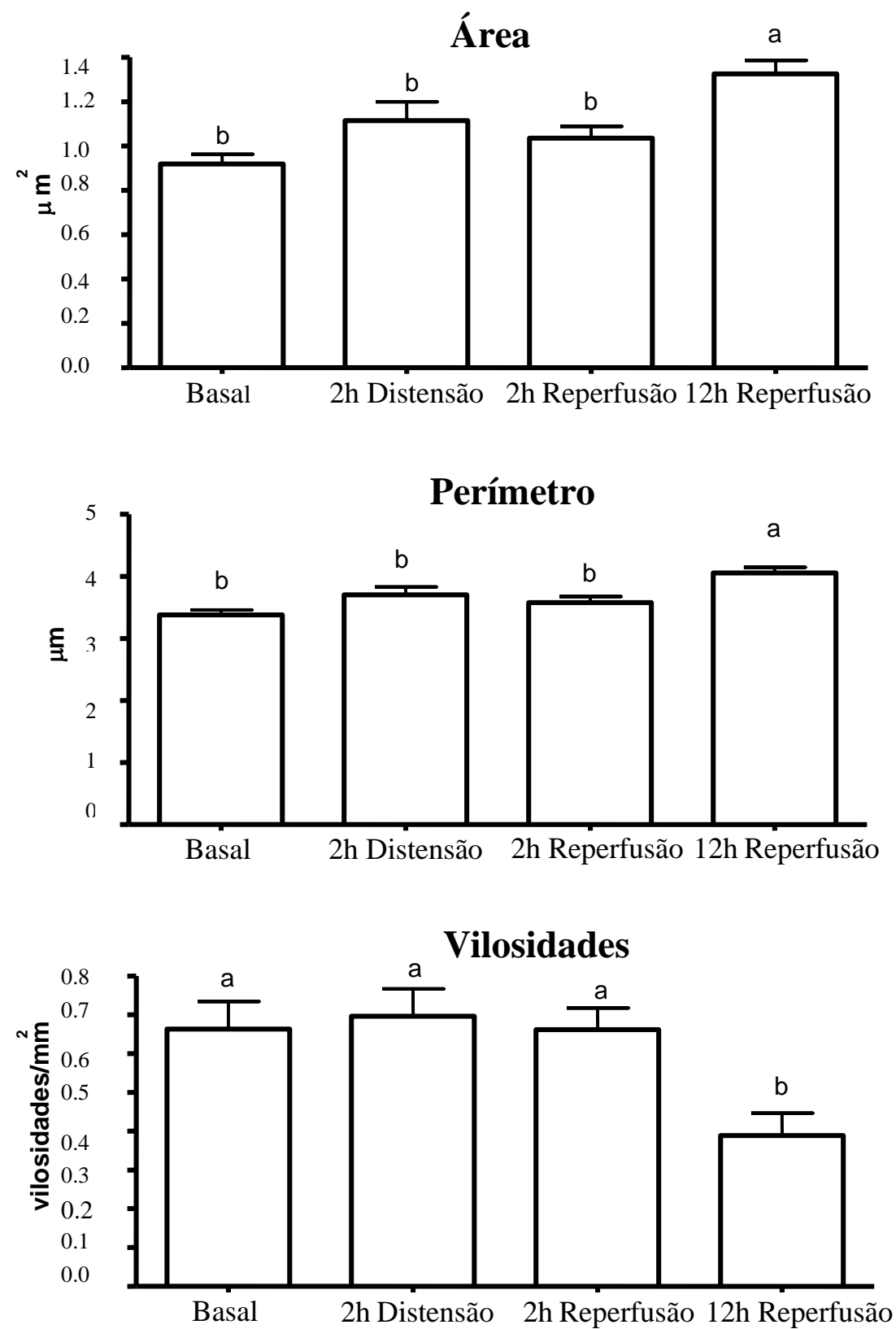

Figura 2. Médias ( \pm erro-padrão) da área, perímetro e densidade das vilosidades intestinais do jejuno equino, antes, ao final da distensão e após duas e $12 \mathrm{~h}$ de reperfusão. 


\section{DISCUSSÃO}

A avaliação por meio da microscopia eletrônica de varredura foi eficaz para verificar as alterações na mucosa do jejuno equino submetido à distensão. Recentemente, Faleiros et al. (2007), ao utilizarem a mesma metodologia, não verificaram alterações evidentes na morfologia do cólon menor equino submetido a modelo de distensão, usando inclusive pressão superior $(40 \mathrm{~mm}$ de $\mathrm{Hg}$ ). Essa discrepância era esperada, pois o estudo revelou que não existe alteração na perfusão da mucosa do cólon menor quando submetida às condições de distensão utilizadas no estudo de Faleiros et al. (2002). Estes achados reforçam observações prévias de que, em equinos, a mucosa do intestino delgado é mais sensível à distensão do que a mucosa do intestino grosso (Dabareiner et al.,1993).

Pelas eletromicrofotografias da mucosa, verificou-se nítida redução do espaço entre as vilosidades nas amostras colhidas ao final da distensão quando comparadas com as do controle. Nesse último estudo, que utilizou o mesmo modelo de distensão no jejuno de equinos, os autores consideraram o inverso, ou seja, aumento desse espaço, baseados na avaliação por microscopia de varredura das réplicas vasculares das vilosidades. Algumas explicações podem ser encontradas para essa discrepância. No presente estudo, que também avaliou numericamente a densidade das vilosidades, tomou-se o cuidado de sempre observar as amostras em um campo de visualização que permitisse analisar integralmente o ápice das vilosidades. Outra possível explicação é que os autores citados não observaram a vilosidade integralmente, mas apenas sua réplica vascular. Assim, não foi possível verificar o achatamento das células epiteliais que recobrem a vasculatura e ocupam o espaço entre as vilosidades.

No presente estudo, duas horas após descompressão, houve o retorno das vilosidades ao formato original. Fato semelhante também foi constatado por Dabareiner et al. (1993), quando observaram que, após a descompressão, a arquitetura das vilosidades voltou a ser semelhante ao estado em que se encontrava antes da distensão. Estes resultados são indicativos de que as alterações vistas após a distensão seriam devido, principalmente, à ação mecânica causada pela solução fisiológica. O fluido, que foi introduzido com o objetivo de manter a pressão em $25 \mathrm{~cm}$, exerceria uma compressão sobre as vilosidades, logo alteraria sua morfologia.

No presente estudo, alterações significativas nas dimensões das vilosidades ocorreram no período de $12 \mathrm{~h}$ após descompressão. Foi observado que, neste tempo, os valores da área e do perímetro das vilosidades foram mais altos $(\mathrm{P}<0,05)$ que os da área e do perímetro de todos os outros tempos (duas horas de isquemia, $12 \mathrm{~h}$ de isquemia e duas horas de reperfusão).

A alteração da morfologia das vilosidades não pode, neste caso, ser explicada apenas pelo fator mecânico da distensão, uma vez que a arquitetura normal já havia sido restabelecida nas primeiras duas horas após a descompressão. $\mathrm{O}$ fato de que as alterações das dimensões das vilosidades ocorreram de forma significativa somente após a descompressão é um indício da presença de lesão inflamatória

Dabareiner et al. (1993) também observaram diminuição da densidade vascular na mucosa e do número de vasos preenchidos na submucosa e mucosa distendidas quando comparado com os resultados dos controles. Desse modo, os achados deste estudo corroboram a hipótese de que o aumento da dimensão das vilosidades seria devido à isquemia e à formação de edema.

A distensão luminal ocasiona compressão dos vasos da parede intestinal, o que promove isquemia. $\mathrm{O}$ edema transmural é causado por aumento na pressão hidrostática capilar, pressão osmótica do plasma diminuída e aumento da permeabilidade capilar (Moore, 1992). Esse aumento só se manifestou depois da descompressão devido à injúria de reperfusão.

Há diversas teorias para explicar os processos que acarretam as lesões de reperfusão. As mais tradicionais estão relacionadas com a ocorrência de microembolismo devido à agregação plaquetária e com o acúmulo de produtos resultantes do metabolismo anaeróbico durante a isquemia. Pesquisas posteriores demonstraram crescente evidência de que a formação de radicais livres está diretamente implicada com os efeitos citotóxicos da reperfusão (Rochat, 1991). 
Durante a isquemia, há conversão da enzima xantina desidrogenase em xantina oxidase e ATP em hipoxantina. Com a reperfusão, ocorre o retorno do oxigênio para o tecido e a formação de radicais de oxigênio, os quais são posteriormente degradados em subprodutos que causam necrose e dano vascular (White, 1990). Alguns autores consideraram que as lesões celulares, observadas durante a reperfusão, podem ser mais graves do que as próprias lesões causadas pela isquemia (Forsyth e Guilford, 1995), e essa consideração condiz com os resultados encontrados no presente estudo.

Além do edema, outro fato que ajudaria a explicar o aumento da dimensão do ápice das vilosidades seria a ocorrência do espaço de Gruenhagen, que é formado no ápice das vilosidades. Ele se localiza entre as células epiteliais e a membrana basal, logo favorece o achatamento das vilosidades, pois tais células perdem a sustentação. Esse espaço já foi verificado em segmentos de jejuno de equinos submetidos a modelos de isquemia e reperfusão (White, 1992) e modelos de distensão e descompressão (Dabareiner et al., 1993).

No tempo de duas horas de reperfusão, não foram constatadas lesões significativas nas vilosidades do jejuno. O fato de as lesões só se manifestarem $12 \mathrm{~h}$ após a descompressão foi, de certa forma, inusitado, uma vez que a consequência da reperfusão na mucosa tem se manifestado predominantemente nos primeiros 180 minutos, conforme verificado no jejuno (Horne et al., 1994; Alves et al., 2003), no cólon maior (Vatistas et al., 1993; Moore et al., 1994) e no cólon menor (Faleiros et al., 2001) de equinos.

Em outro modelo experimental para estudo da regeneração do epitélio do jejuno submetido a períodos de duas e três horas de obstrução estrangulativa hemorrágica ou isquêmica, verificou-se que, em determinados segmentos, houve comprometimento da mucosa nas primeiras $12 \mathrm{~h}$ de reperfusão (Freeman et al., 1988). De modo geral, considerou-se que a injúria de reperfusão se inicia nos primeiros dois minutos de reperfusão e pode durar horas ou dias (White, 1995).

Supõe-se que as alterações nas vilosidades foram mais acentuadas após a descompressão devido às lesões promovidas por isquemia e reperfusão da mucosa intestinal. Entretanto, não foi possível explicar o resultado encontrado de que as alterações nas dimensões das vilosidades se manifestaram apenas $12 \mathrm{~h}$ após a reperfusão e que nada ocorreu duas horas após a descompressão.

\section{CONCLUSÕES}

A distensão luminal do jejuno de equinos, com solução salina a uma pressão de $25 \mathrm{~cm}$ de $\mathrm{H}_{2} \mathrm{O}$, por período de duas horas, promove aumento tardio das dimensões das vilosidades intestinais. Observam-se alterações nas vilosidades no tempo de $12 \mathrm{~h}$ de reperfusão e nada é observado no tempo de duas horas de descompressão.

\section{AGRADECIMENTOS}

À Fundação de Amparo a Pesquisa do Estado de Minas Gerais (FAPEMIG), pelo apoio financeiro por meio do projeto - CVZ 23704-.

\section{REFERÊNCIAS BIBLIOGRÁFICAS}

ALVES, G.E.S.; MATOS, J.J.R.T.; FALEIROS R.R. et al. Efeito da hidrocortisona sobre a lesão de reperfusão e reparação da mucosa após isquemia venosa experimental no jejuno de equinos. Arq. Bras. Med. Vet. Zootec., v.55, p.539-549, 2003.

DABAREINER, R.M.; SULLINS, K.E.; SNYDER, J.R. et al. Evaluation of the microcirculation of the equine small intestine after intraluminal distension and subsequent decompression. Am. J. Vet. Res., v.54, p.16731681, 1993.

DABAREINER, R.M.; SULLINS, K.E.; WHITE, N.A. et al. Serosal injury in the equine jejunum and ascending colon after ischemiareperfusion or intraluminal distention and decompression. Vet. Surg., v.30, p.114-125, 2001.

FALEIROS, R.R.; ALVES, G.E.S.; SANTOS R. et al. Experimental ischemia and reperfusion in equine small colon. Arq. Bras. Med. Vet. Zootec., v.53, p.341-350, 2001

FALEIROS, R.R.; MACORIS, D.G.; ALVES, G.E.S. et al. Avaliação histomorfométrica e ultraestrutural da mucosa do cólon menor equino submetido a distensão, Pesq. Vet. Bras., v.27, p.381-385, 2007. 
FALEIROS, R.R.; MACORIS, D.G.; ALESSI A.C. et al. Effect of intraluminal distension on microvascular perfusion in the equine small colon. Am. J. Vet. Res. v.63, p.1292-1297, 2002.

FORSYTH, S.F.; GUILFORD, W.G. Ischaemia reperfusion injury - a small animal perspective. Br. Vet. J., v.151, p.281-198, 1995.

FREEMAN, D.E.; CIMPRICH, R.E.; RICHARDSON, D.W. et al. Early mucosal healing and chronic changes in pony jejunum after various types of strangulation obstruction. Am. Vet. Res., v.49, p.810-818, 1988.

HORNE, M.M.; PASCOE, P.J.; DUCHARME, N.G. et al. Attempts to modify reperfusion injury of equine jejunal mucosa using dimethylsulfoxide, allopurinol, and intraluminal oxygen. Vet. Surg., v.4, p.241-249, 1994.

MOORE, R.M. Pathophysiology of intestinal ischemia and endotoxemia. Equine Prac., v.14, p.13-15, 1992.

MOORE, R.M.; MUIR, W.W.; BERTONE, A.L. et al. Characterization of the hemodynamic and metabolic alterations in the large colon of horses during low-flow ischemia and reperfusion. Am. J. Vet. Res., v.55, p.1444-1453, 1994.

ROCHAT, M.C. An introduction to reperfusion injury. Compend. Contin. Educ. Pract. Vet., v.13, p.923-930, 1991.
SAMPAIO, I.B.M. Estatística aplicada à experimentação animal. Belo Horizonte: FEPMZV, 1998. 221p.

SPEIRS, C.V. Clinical examination of horses. Pennsylvania: Saunders, 1997. 358p.

THOMASSIAN, A. Enfermidades dos cavalos. 2.ed. São Paulo: Varela, 1990. 561p.

VATISTAS N.J., SNYDER J.R., HILDEBRAND S.V. et al. Effects of the 21aminosteroid U-74389G, on ischemia and reperfusion injury of the ascending colon in horses. Am. J. Vet. Res., v.54, p.2155-2160, 1993.

WHITE, N.A. Epidemiology and etiology of colic. In: WHITE, N.A. The equine acute abdomen. Philadelphia: Lea \& Febiger, 1990. p.49-64.

WHITE, N.A. Conditions of the small intestine. Equine Pract., v.14, p.16-21, 1992.

WHITE, N.A. Alterations of the intestines during obstruction and ischemia. In: CICLO INTERNACIONAL DE CÓLICA EQUINA, 2.,1995, Jaboticabal. Anais... Jaboticabal: UNESP, 1995. p.39-50. 